
\title{
3 Research Suare \\ Identification of Small Compounds Regulating the Secretion of Extracellular Vesicles via A TIM4- Affinity ELISA
}

\section{Yunfei Ma}

Kanazawa University

Takeshi Yoshida

Kanazawa University

Kazutaka Matoba

Nissan Chemical Corporation (Japan)

Katsuhiko Kida

Nissan Chemical Corporation (Japan)

Rito Shintani

Kanazawa University

\section{Yingshi Piao}

Yanbian University

Jingchun Jin

Yanbian University Hospital

Taito Nishino

Nissan Chemical Corporation (Japan)

Rikinari Hanayama ( $\nabla$ rikinari-hanayama@umin.ac.jp )

Kanazawa University

\section{Research Article}

Keywords: extracellular vesicle, high-throughput screening, chemical library, ELISA, angiogenesis, apoptosis

Posted Date: February 3rd, 2021

DOI: https://doi.org/10.21203/rs.3.rs-151218/v1

License: (c) (1) This work is licensed under a Creative Commons Attribution 4.0 International License.

Read Full License 


\section{Abstract}

Extracellular vesicles (EVs) are secreted from most cells and play important roles in cell-cell communication by transporting proteins, lipids, and nucleic acids. As the involvement of EVs in diseases has become apparent, druggable regulators of EV secretion are more desirable. However, the lack of a highly sensitive EV detection system has made the development of EV regulators difficult. We developed an ELISA system to detect EVs using TIM4 proteins, which have high affinity to phosphatidylserine and screened a 1,567-compound library. Consequently, we identified one inhibitor and three activators of EV secretion in a variety of cells. The inhibitor, apoptosis activator 2, suppressed EV secretion via a different mechanism and had a broader cellular specificity than GW4869. The three activators, cucurbitacin B, gossypol, and obatoclax, also had broad cellular specificity, including HEK293T cells and human mesenchymal stem cells (hMSCs). In vitro bioactivity assays revealed that some regulators control EV secretion from glioblastoma and hMSCs, which induces angiogenesis and protects cardiomyocytes against apoptosis, respectively. In conclusion, we developed a high-throughput method to detect EVs with high sensitivity and versatility, and identified three compounds that can regulate the bioactivity of EVs.

\section{Introduction}

Nano-sized extracellular vesicles (EVs), including exosomes, are released from most cells and play important roles in cell-cell communication by transporting proteins, lipids, and nucleic acids. EVs have been studied widely in recent decades due to their involvement in various physiological phenomena and pathological processes, including adaptive immune responses, neurodegenerative diseases, and tumor progression ${ }^{1}$. In particular, tumor-derived EVs promote tumor metastasis by altering the tumor microenvironment ${ }^{2,3}$, induce angiogenesis by directly or indirectly activating the vascular endothelial cells $^{4-6}$, and suppress antitumor immunity by activating immune checkpoints ${ }^{7}$. Suppressing the effects of EVs may be a potential therapeutic strategy for several EV-related diseases ${ }^{8}$. EVs have also attracted attention as therapeutic agents. For example, EVs derived from mesenchymal stem cells (MSCs) have been shown to have a therapeutic effect on neurodegenerative diseases ${ }^{9}$. Engineered-EVs artificially loaded with effective proteins, nucleic acids, or small compounds have also been developed ${ }^{10}$. Therapies targeting or utilizing EVs have potential in many diseases; however, their clinical use has been limited by a delay in the development of EV regulators that can modulate EVs without inducing severe side effects.

To date, several compounds have been reported to regulate EV secretion. Monensin, A23187, and ionomycin can facilitate EV release by increasing the concentration of intracellular calcium ions ${ }^{12-14}$. GW4869, an inhibitor of neutral sphingomyelinase 2 (nSMase2, or SMPD3), suppresses EV secretion by inhibiting the development of ceramide-dependent multivesicular endosome (MVE) ${ }^{15}$. Bafilomycin, and other autophagy inhibitors targeting V-ATPase, can markedly increase EV secretion ${ }^{16,17}$. However, cells generate and secrete EVs via multiple pathways. Development of MVEs is mediated by at least three

pathways: ubiquitin-dependent endosomal sorting complex required for transport (ESCRT) pathway ${ }^{18,19}$, ubiquitin -independent ESCRT pathway ${ }^{20}$, and ESCRT-independent pathway ${ }^{15}$. MVE trafficking is 
mediated by multiple Rabs, including Rab11, Rab27, and Rab35 ${ }^{21}$. EV secretion caused by fusion of MVEs and plasma membrane is mediated by SNARE and MUNC13-4 22,23 . Therefore, the existing regulators are not enough to regulate EV secretion in multiple cell types studied in basic or clinical studies. Some studies have screened EV regulators by using cells overexpressing fusion proteins of exosome marker and reporter proteins, such as CD63-EGFP or CD63-nanoluciferase, to increase the EV signal ${ }^{17,24}$, because marker proteins loaded on EVs are difficult to be detected in high-throughput screens. However, overexpression of fusion proteins may affect the physiological function, and the screened compounds may regulate CD63-positive EVs but not all EVs.

TIM4 binds to phosphatidylserine (PS) with high affinity $\left(\mathrm{K}_{\mathrm{d}} \approx 2 \mathrm{nM}\right)$ and was identified as a receptor involved in the uptake of $\mathrm{EVs}^{25}$. As PS is enriched in $\mathrm{EVs}^{26}$, we previously developed an efficient method of isolating EVs using TIM4-affinity beads ${ }^{27}$. In this study, we employed a TIM4 EV-ELISA system, which enabled the detection of native EVs with high sensitivity using multiple antibodies against EV markers. Furthermore, a drug-repositioning library containing compounds investigated in phase I clinical trials was screened to identify druggable EV regulators. Consequently, we identified several compounds regulating EV secretion, and showed that some are able to regulate the biological activities of EVs.

\section{Results}

The TIM4-ELISA screen identified nine EV-regulators from a library of 1567 compounds. TIM4 binds to PS with high affinity, and has demonstrated the ability to capture EVs, because most EVs expose PS on their surfaces $^{27}$. Here, we applied TIM4 to a sandwich ELISA system for a high-throughput chemical screen. The TIM4-ELISA showed good quantitative capability (Fig. $1 \mathrm{a}, \mathrm{CV}=8.25$ and $3.46 \%$ at 0 and $10 \mu \mathrm{M}$, respectively, $S / N=8.70, R^{2}=0.9832$ ) in an experiment of monensin-dependent EV secretion ${ }^{11}$. We prepared a drug-repositioning library containing 1567 compounds, and screened EV regulators using the TIM4-ELISA by treating U87MG cells with three doses of each drug $(0.1,1$, and $10 \mu \mathrm{M})$. A compound increasing the secretion of EVs by more than 1.5 -fold or less than 0.67 -fold was classified as an activator or inhibitor, respectively. After the first round of screening using the TIM4-CD63 ELISA, 38 and 22 compounds remained candidate EV activators and inhibitors, respectively (Fig. 1b). In the second round of screening, we examined the effects of the compounds on EV secretion using TIM4-CD9 and TIM4CD63 ELISAs and evaluated the cytotoxicity of the compounds. Consequently, 18 and six compounds were able to induce or inhibit EV secretion without obvious cytotoxicity. In the third round of screening, in addition to the previous assessments, we also evaluated the concentration of EV particles by nanoparticle tracking analysis (NTA). Consequently, we identified an inhibitor, apoptosis activator 2 (AA2) (Table 1), which induced a 66.4 and $45.3 \%$ decrease in the secretion of CD9- and CD63-positive EVs, respectively, and a $35.1 \%$ decrease in the number of secreted EVs without affecting cell growth (Fig. 1cf). We also identified eight EV activators: amlodipine, osimertinib, cucurbitacin B, doramectin, gossypol, HA14-1, miltefosine, and obatoclax (Table 1). These compounds induced a 1.3- to 5.7-fold increase in the secretion of CD9- or CD63-positive EVs without affecting the growth of U87MG cells (Fig. $1 \mathrm{~g}-\mathrm{i}$ ). NTA 
revealed that amlodipine, cucurbitacin B, doramectin, gossypol, miltefosine, and obatoclax were able to increase the number of EV particles (Fig. 1j).

AA2 inhibited EV secretion independent of nSMase2 and caspase 3. To investigate the structure-activity relationship of AA2, we evaluated the effects of 10 AA2 analogs (Table 2). Five analogs, (\#2, \#4, \#5, \#6, and \#8) moderately affected EV secretion in TIM4-CD9, TIM4-CD63 ELISA, and NTA (Fig. 2a, b). AA2 and these effective compounds possessed two chlorines at the 3,4 position of the benzyl group or carried no halogen in the benzyl group. Notably, even the simplest analog (\#8) demonstrated moderate activity. Modifications around the indole ring resulted in slight changes in the activity of the analogs. While compounds $1, \# 3, \# 7, \# 9$, and $\# 10$, which presented no activity, possessed one halogen in the benzyl group or two chlorines at the 2,6 position of the benzyl group. These data indicated that the 3,4dichlorobenzyl group in AA2 is essential for the inhibitory effect on EV secretion. Next, we evaluated the effect of AA2 on different human and mouse cell lines. Consequently, AA2 inhibited the secretion of EVs in eight of nine cell lines, namely THP-1, SW620, U87MG, SW480, 293T, HCT116, Jurkat, and EL4 (Fig. 2C). A similar evaluation of GW4869, an inhibitor of EV secretion targeting nSMase ${ }^{15}$, revealed that EV secretion was inhibited in four of nine cell lines, namely NIH/3T3, Jurkat, EL4, and THP-1 (Fig. 2d). As the cellular specificity of AA2 and GW4869 differ, AA2 may inhibit a pathway other than the nSMase2dependent pathway.

AA2 is a strong activator of caspase 3 , which plays a key role in the execution phase of apoptosis ${ }^{28}$. However, in our study, AA2 inhibited EV secretion at concentrations lower than those that induced cell death (Fig. 1c-f). Thus, AA2 may suppress EV secretion independent of caspase 3 -activation. To test this hypothesis, we evaluated the effect of MT-21, an activator of caspase 3, on EV secretion and showed that MT-21 failed to inhibit EV secretion without affecting cell growth (Fig. 2e-g). Furthermore, we used an irreversible pan-caspase inhibitor Q-VD-OPH or CASP3 knock-down (KD) cells to exclude the involvement of caspase 3. Q-VD-OPH inhibited the cytotoxicity of high-concentrations of AA2 and restored the growth of Jurkat cells (Fig. 2h, i). Western blotting analysis for caspase 3-cleavage also showed that Q-VD-OPH inhibited caspase 3 activation induced by AA2 (Fig. 2j). Conversely, treatment with Q-VD-OPH did not affect the inhibitory effect of AA2 on EV secretion (Fig. 2k). Consistent results were obtained in treatment with another caspase 3 inhibitor, Z-VAD(OMe)-FMK, although it was less effective than Q-VD-OPH in recovering cell growth (Supplementary Fig. S1a-c). Similarly, AA2 inhibited EV secretion to a similar level in both HEK293T wild-type and CASP3-KD cells (Fig. 2I-n). These data indicated that the inhibition of EV secretion by $A A 2$ is independent of caspase 3 -activation.

Cucurbitacin B, gossypol, and obatoclax induce EV secretion in a variety of cell lines. HEK293T and HEK293 cells are commonly used to prepare engineered $\mathrm{EVs}^{29-32}$. We evaluated the effect of eight EV activators on CD63-positive EV secretion in these cells. After determining the nontoxic concentration of each activator, cells were treated at the maximum non-toxic concentration. Cucurbitacin B, gossypol, and obatoclax induced CD63-positive EV secretion in both HEK293T and HEK293 cells (Fig. 3a,

Supplementary Fig. S2a). These three activators also promoted the secretion of CD9- and CD63-positive EVs and increased the number of EV particles secreted from HEK293T and HEK293 cells (Fig. 3b, c and 
Supplementary Fig. S2a, b). Next, we tested these three compounds in a variety of cells. We found that cucurbitacin B induced EV secretion in all cells except for Jurkat cells, showing broad cellular specificity (Fig. 3d). Gossypol and obatoclax presented a slightly narrower cellular specificity. (Fig. 3d). No compounds induced EV secretion in Jurkat cells. These data indicated that the effect of activators on EV secretion is cell-type dependent.

\section{EV regulators have potential to regulate the bioactivity of EVs. Previous studies have demonstrated the} contribution of tumor-derived EVs to angiogenesis in tumor progression ${ }^{6,33-35}$. In particular, glioblastomaderived EVs containing WT1 protein are transferred into microglia, where they suppress the expression of thrombospondin 1 (Thbs1), a negative regulator of angiogenesis ${ }^{6,36}$. To clarify whether the identified EV regulators have the potential to regulate EV bioactivity, we assessed their effects on the induction of angiogenesis by glioblastoma-derived EVs. As expected, EVs from U87MG cells treated with DMSO downregulated expression of Thbs1 in microglia MG6 cells, while those treated with AA2 recovered the expression of Thbs 1 in treatment of the EVs from the same number of cells (Fig. 4a). Conversely, the effect on Thbs 1 expression was comparable between the two groups in treatment of the same particle number of the EVs (Fig. 4b). Similarly, cucurbitacin B and obatoclax enhanced the effect of EVs when the EVs from the same number of cells were treated, and had little or no change in the effect when the same number of particles were treated (Fig. 4c, d). These data indicated that AA2, cucurbitacin B, and obatoclax can regulate the number of secreted EVs maintaining their quality. Additionally, we evaluated the ability of these $E V$ regulators to protect cardiomyocytes against hypoxia-induced apoptosis by hMSC-derived $\mathrm{EVs}^{37}$. EVs from hMSCs treated with DMSO did not recover the growth of cardiomyocyte H9C2 cells, while those treated with gossypol or obatoclax significantly recovered the growth of $\mathrm{H} 9 \mathrm{C} 2$ cells when the EVs from the same number of cells were treated (Fig. 4e). These results suggested that the EV regulators may have therapeutic potential for EV-related diseases.

\section{Discussion}

In this study, we screened compounds that can regulate EV secretion using a TIM4-affinity EV-ELISA system. Compared with the traditional EV-ELISA using anti-CD63 antibodies and so on, the TIM4-ELISA enables EVs to be captured with low-bias, and with10-fold higher sensitivity ${ }^{27}$. These characteristics are useful for high-throughput screenings on a small-scale, such as those performed in a 384-well plate, or detection following a short stimulation of no more than $24 \mathrm{~h}$. In our study, we identified one inhibitor and eight activators by screening a 1,567-compound library. The inhibitor, AA2, inhibited EV secretion from a variety of cells in vitro, including tumor and non-tumor cells. However, considering the effect of AA2 on apoptosis, its use clinically for patients with EV-related diseases may be limited. Notably, the inhibitory effect of AA2 on EV secretion was observed with concentrations lower than those that induce apoptosis in most cells, and EV secretion was inhibited via a pathway independent of caspase 3 . Therefore, it may be possible to develop an inhibitor that regulates EV secretion with no or limited effects on apoptosis. We were unable to identify such a compound in this study; however, we elucidated the structure-activity relationship of AA2 analogs. This information will be helpful for developing suitable AA2 derivatives. 
To characterize the effects of AA2, we determined its cellular specificity by comparing with GW4869, an inhibitor of exosome secretion that targets nSMase2. Consequently, AA2 was found to possess different and wider cellular specificity than GW4869. This indicated that AA2 inhibits EV secretion via a pathway independent of nSMase2. Based on the NCBI Gene database (https://www.ncbi.nlm.nih.gov/gene/), NSMASE2 mRNA is expressed in limited human and mouse tissues. We hypothesized that the narrow cellular specificity of GW4869 may be due to the limited expression of NSMASE2. Conversely, AA2 has wider cellular specificity, suggesting that AA2 may target a molecule expressed in various cell types. Overall, the different cellular specificities of AA2 and GW4869 might be helpful for inhibiting EV secretion in a variety of cells.

In the last decade, EVs have aroused attention as a drug delivery tool for bioactive proteins or nucleic acids. Basic and clinical studies have attempted to develop engineered-EVs or MSC-derived EVs for tumor therapy, immune activation, and wound repair. Because HEK293T cells and human MSCs are often used to produce EVs, there has been a desire for small compounds that can induce EV secretion from these cells. Datta et al. identified EV activators, including fenoterol, norepinephrine, N-methyldopamine, mephenesin, and forskolin, after screening a chemical library in prostate cancer cell lines ${ }^{24}$; however, these compounds did not have the desired effect in human MSCs ${ }^{38}$. Three of eight compounds which we identified from the screen in U87MG cells, including gossypol, obatoclax, and cucurbitacin B, also induced EV secretion from HEK293T cells, HEK293 cells, and human MSCs. As we demonstrated that these activators differ in their cellular specificity, it is important to select the most effective activator for each cell type. To use these activators to produce bioactive EVs, the biological activity of EVs produced following treatment should be no less than that of EVs produced without treatment. Evaluation of Thbs1 mRNA regulation by glioma-derived EVs revealed that the activators increased the effects of glioma-EVs when the EVs from the same number of cells were treated, while they were affected at similar or higher levels when the same number of particles were treated. Thus, the activators can increase the number of EVs secreted from cells without changing their quality, and therefore possess the desired properties for producing EVs.

Notably, cucurbitacin B is a Bcl-2, Mcl-1, and Bcl-XL inhibitor ${ }^{39}$, and gossypol and obatoclax are pan-Bcl-2 family inhibitors ${ }^{40}$. These three compounds induce apoptosis by inhibiting members of the anti-apoptotic $\mathrm{Bcl}-2$ family but are not known to regulate EV secretion. Members of the anti-apoptotic Bcl-2 family repress apoptosis by inhibiting $\mathrm{Ca}^{2+}$ release from the endoplasmic reticulum (ER), in so-called calcium induced apoptosis ${ }^{41}$. As the involvement of $\mathrm{Ca}^{2+}$ signaling in $\mathrm{EV}$ secretion has been reported in several cell lines ${ }^{13,23}$, it is possible that the release of $\mathrm{Ca}^{2+}$ from ER by the activators may induce EV secretion. Treatment with these activators at non-toxic concentrations may induce $\mathrm{Ca}^{2+}$ release at levels not sufficient to induce apoptosis, but sufficient to induce EV secretion. In addition, autophagy induced by these activators may enhance EV secretion, since members of the anti-apoptotic Bcl-2 family are autophagy suppressors ${ }^{42}$. Autophagosomes generally develop into autolysosome by fusing with lysosomes; however, by inhibiting this pathway, amphisomes are developed by fusing the accumulated 
autophagosomes with MVBs, which are then exported from the cells. The activators enhance the generation of autophagosomes and amphisomes, which may indirectly increase EV secretion.

Here, we identified several EV regulators through high-throughput screening of a 1567-compound library. Capture of EVs by a high-affinity lipid binder, and detection of EVs using multiple EV surface markers, enabled the efficient identification of EV regulators. The EV regulators rarely change the quality of EVs but regulate the number of EVs secreted, presenting the desired characteristics for drug development. We tested the effects of EV regulators only in vitro, thus there are still several issues to be verified before they are used for the treatment of EV-related diseases, including the delivery system of EV regulators to target cells, effects on EV secretion from normal cells, and side effects. We hope that these regulators will contribute to the development of EV drugs or the therapy for EV-related diseases by resolving the issues.

\section{Methods}

Cells. U87MG (ATCC, Manassas, VA), HEK293 (Riken BRC, Ibaraki, Japan), HEK293T (Riken BRC), NIH/3T3 (ATCC), LM8 (Riken BRC), HCT116 (Riken BRC), SW480 (ATCC), SW620 (ATCC), EL4 (Riken BRC), and MG6 (Riken BRC) cell lines were maintained in Advanced DMEM (Thermo Fisher Scientific, Waltham, MA) supplemented with $2 \%$ heat-inactivated fetal bovine serum (FBS; Thermo Fisher Scientific), penicillin-streptomycin, and L-glutamine. K562 (Riken BRC), Jurkat (Riken BRC), and THP-1 (Riken BRC) cells were maintained in Advanced RPMI-1640 (Thermo Fisher Scientific) with 2\% heat-inactivated FBS, penicillin-streptomycin, and L-glutamine. Human mesenchymal stem cells from adipose tissue (PromoCell, Heidelberg, Germany) were maintained in Cellartis MSC Xeno-Free Culture Medium (Takara Bio, Shiga, Japan) with Cellartis MSC Xeno-Free Supplement, and used in experiments by maximum 10 passages. H9C2 (ATCC) cells were maintained in high-glucose DMEM (Thermo Fisher Scientific) supplemented with $10 \% \mathrm{FBS}$, penicillin-streptomycin, and L-glutamine. All cells were cultured at $37^{\circ} \mathrm{C}$ with $5 \% \mathrm{CO}_{2}$. To detect EVs, FBS was replaced with EV-free FBS, which was prepared by mixing $10 \mathrm{~mL}$ of heatinactivated FBS with $2 \mathrm{~mL}$ of $50 \%$ PEG-10,000 (Merck, Darmstadt, Germany) by rotating at $4^{\circ} \mathrm{C}$ for $4 \mathrm{~h}$. The supernatant was then collected after centrifugation at 2,000 $\times g$ for $20 \mathrm{~min}$. HEK293T CASP3 KD cells were established via the CRISPR/Cas9 system. In brief, a pX330 plasmid targeting a sequence in the human CASP3 gene (GGAATGACATCTCGGTCTGG, PAM sequence is underlined) was transfected into HEK293T cells. After 3 days of selection with puromycin, CASP3 expression was determined via western blot analysis. All cell lines were tested for mycoplasma contamination by PCR targeting the 16S ribosome.

Chemical treatment. U87MG cells were seeded in Advanced DMEM-2\% EV-free FBS containing $0.03 \%$ SphereMax (Nissan chemical, Tokyo, Japan) into 384-well plate or plates of other sizes, and treated with a chemical library, ionomycin (Merck), monensin (Merck), or DMSO (Merck). Other cells were seeded in Advanced medium-2\% EV-free FBS into 96- or 24-well plates, and treated with a chemical library, ionomycin, monensin, or DMSO. After incubating the cells for $24 \mathrm{~h}$, the plate was centrifuged at 1,200 $\times g$ for 60 min to separate the conditioned medium (1.2K sup) from the cells. A WST-8 assay (Nacalai Tesque, Kyoto, Japan) was used to evaluate cellular proliferation, according to the manufacturer's protocols. To 
evaluate the amount of EVs or cytotoxicity, the 1.2K sup was subjected in TIM4-affinity ELISA or LDH assay. The LDH assay (Dojindo, Kumamoto, Japan) was performed according to the manufacturer's protocol. In experiments performed on a larger than 384-well scale, the conditioned medium was separated by serial centrifugation at $300 \times g$ for $5 \mathrm{~min}, 2,000 \times g$ for $20 \mathrm{~min}, 10,000 \times g$ for $30 \mathrm{~min}$, and was then (10K sup) used in TIM4-affinity ELISA, NTA, or for the isolation of EVs.

TIM4-affinity ELISA. An ELISA plate (AGC Techno Glass, Shizuoka Japan) was coated with $1 \mu \mathrm{g} / \mathrm{mL}$ recombinant mouse TIM4-Fc protein/50 mM carbonate-bicarbonate buffer ( $\mathrm{pH}$ 9.6) (FUJIFILM Wako Pure Chemical, Osaka, Japan) and incubated at $4^{\circ} \mathrm{C}$ overnight. The wells were blocked with $1 \%$ bovine serum albumin (BSA) $/ 2 \mathrm{mM} \mathrm{CaCl} / 2 / 0.05 \%$ tween20/TBS buffer (TBS-TCa) for $1 \mathrm{~h}$. Then, $1.2 \mathrm{~K}$ sup or $10 \mathrm{~K}$ sup supplemented with $2 \mathrm{mM} \mathrm{CaCl}_{2}$ was applied to the wells, and the cells were incubated for $1 \mathrm{~h}$ at room temperature. The captured EVs were labeled with each primary antibody/TBS-TCa for $2 \mathrm{~h}$ and then each HRP-conjugated secondary antibody for $1 \mathrm{~h}$ at room temperature. Finally, EVs were detected with TMB reagent (Nacalai Tesque) by measuring the absorbance at $450 \mathrm{~nm}$. Primary antibodies against mouse $\mathrm{CD} 9$, mouse $\mathrm{CD} 63$, mouse/rat CD81, human CD9, human CD63, and human CD81 were purchased form Biolegend. HRP-conjugated secondary antibodies against rat IgG, Armenian hamster IgG, and mouse IgG were purchased from BioLegend, Jackson ImmunoResearch, and BioLegend, respectively.

Western blotting. Cells were lysed in RIPA buffer [50 mM Tris-HCl (pH 8.0), $150 \mathrm{mM} \mathrm{NaCl}, 2 \mathrm{mM}$ EDTA, $0.5 \%$ sodium deoxycholate, $1 \%$ TritonX- $100,0.1 \%$ sodium dodecyl sulfate] with protease inhibitor cocktail on ice for $20 \mathrm{~min}$, and then centrifuged at $14,000 \times \mathrm{g}, 4^{\circ} \mathrm{C}$ for $20 \mathrm{~min}$ to harvest the cell lysates. The cell lysates were separated by SDS-PAGE and transferred onto PVDF membranes. The membranes were blocked in $5 \%$ skim milk in $0.05 \%$ tween $20 /$ TBS buffer for $1 \mathrm{~h}$, and then incubated with each primary antibody at $4^{\circ} \mathrm{C}$ overnight, followed by each HRP-conjugated secondary antibody. Immunoblot signals were captured using the Image Quant Las 4000mini (GE Healthcare, Chicago, IL) and SuperSignal West Pico Chemiluminescent Substrate (Thermo Fisher Scientific). Primary antibodies against caspase 3 and cleaved caspase 3 were purchased from Cell Signaling Technology (Danvers, MA), while those against $\beta$ actin were purchased from Merck. HRP-conjugated secondary antibodies against rabbit and mouse IgG were purchased from BioLegend.

NTA. The $10 \mathrm{~K}$ sup was diluted to an appropriate concentration in PBS (-), and then the concentration of extracellular vesicles was determined using NanoSight LM10 (Malvern Panalytical, Malvern, United Kingdom). The movement of EVs was recorded three times at the same temperature for $30 \mathrm{~s}$, and then analyzed using the NTA3.1 software.

Model of glioblastoma-derived EV-mediated angiogenesis. EVs were isolated from the 10K sup of U87MG cells following treatment for 2 days with DMSO or EV regulators, using the MagCapture ${ }^{\mathrm{TM}}$ Exosome Isolation Kit PS (FUJIFILM Wako Pure Chemical) according to the manufacturer's instructions. After isolating EVs, the buffer was replaced with PBS (-) in dialysis. The effect of U87MG-EVs was evaluated in a model of EV-mediated angiogenesis, as previously reported ${ }^{43}$. Briefly, MG6 cells were seeded at $1 \times 10^{5}$ cells in a 24-well plate and cultured for $12 \mathrm{~h}$. The cells were treated for $12 \mathrm{~h}$ with the indicated amounts of 
U87MG-EVs. After washing the cells with PBS (-), total RNA was extracted using RNeasy Plus Mini Kits (QIAGEN, Hilden, Germany). Single-strand cDNA was synthesized using ReverTra Ace qPCR master mix (TOYOBO, Osaka, Japan). Thbs1 or Gapdh gene was amplified and detected using the LightCycler 96 (Roche, Basel, Switzerland) with Universal SYBR Select master mix (Thermo Fisher Scientific) and paired primers, Thbs7-Fw; 5'-CACCTCTCCGGGTTACTGAG-3' and Thbs 1-Rv; 5'-GCAACAGGAACAGGACACCTA-3', or Gapdh-Fw; 5'- GTGTTTCCTCGTCCCGTAGA-3" and Gapdh-Rv; 5'-AATCTCCACTTTGCCACTGC-3'.

Hypoxia-induced cardiomyocyte apoptosis assay. To isolate EVs, $4 \times 10^{5} \mathrm{hMSC}$ were cultured for 4 days and then treated for 2 days with DMSO or EV regulators. The EVs were isolated from $10 \mathrm{~K}$ sup of the hMSC-conditioned medium using the Capturem ${ }^{\text {TM }}$ Exosome Isolation Kit (Takara Bio), according to the manufacturer's instructions. After isolating EVs, the buffer was replaced with PBS (-) using the Amicon Ultra PLGC Ultracel column, 10 membrane, $3 \mathrm{kDa}$ column (Merck). To induce hypoxia-induced apoptosis, $5 \times 10^{4} \mathrm{H} 9 \mathrm{C} 2$ cells were cultured for 1 day in DMEM-10\%FBS, followed by 1 day in DMEM- $10 \% \mathrm{FBS}$ containing $1 \mathrm{mM} \mathrm{CoCl}_{2}$ (FUJIFILM Wako Pure Chemical), and 4 days in DMEM-10\%FBS containing the EVs from hMSCs treated with DMSO or EV regulators. The cells were used to perform a in CellTiter-Glo Luminescent Cell Viability Assay (Promega, Madison, WI) according to the manufacture's protocols.

\section{Declarations}

\section{Acknowledgements}

We thank Y. Okayasu for secretarial assistance. This work was supported by Core Research for Evolutional Science and Technology (CREST) from Japan Science and Technology Agency (JST) (No. JPMJCR18H4 to R.H.), Grants-in-Aid for Scientific Research (KAKENHI) from the Ministry of Education, Culture, Sports, Science and Technology (MEXT) (No. $19 \mathrm{H} 03433$ to R.H.), National Natural Science Foundation of China (No. 81860651 to Y.P.; No. 31460294 to J.J.) and projects funded by the Science and Technology Department of Jilin Province (No. 20200201492JC to J.J.), and the World Premier International Research Center Initiative (WPI), MEXT, Japan.

\section{Author contributions}

T.Y., K.M., T.N. and R.H. designed the research; Y.M., T.Y., K.M, K.K., R.S., Y.P. and J.J. performed the experiments and analyzed the data; Y.M., T.Y., T.N. and R.H. wrote the paper.

\section{Competing interests}

The authors declare no competing interests.

\section{Data Availability}

The authors declare that all data supporting the findings of this study are available within the paper and the associated supplementary information files, or from the corresponding author upon reasonable request. 


\section{References}

1. Yáñez-Mó, M. et al. Biological properties of extracellular vesicles and their physiological functions.J. Extracell. Vesicles4, (2015).

2. Hood, J. L., San, R. S. \& Wickline, S. A. Exosomes Released by Melanoma Cells Prepare Sentinel Lymph Nodes for Tumor Metastasis. Cancer Res. 71, 3792-3801 (2011).

3. Peinado, H. et al. Melanoma exosomes educate bone marrow progenitor cells toward a prometastatic phenotype through MET. Nat. Med. 18, 883-891 (2012).

4. Ko, S. Y. et al. Cancer-derived small extracellular vesicles promote angiogenesis by heparin-bound, bevacizumab-insensitive VEGF, independent of vesicle uptake. Commun. Biol. 2, 386 (2019).

5. Wang, Y. et al. Tumor-Derived EV-Encapsulated miR-181b-5p Induces Angiogenesis to Foster Tumorigenesis and Metastasis of ESCC. Mol. Ther. - Nucleic Acids. 20, 421-437 (2020).

6. Tsutsui, T. et al. Glioma-derived extracellular vesicles promote tumor progression by conveying WT1. Carcinogenesis bgaa052(2020) doi:10.1093/carcin/bgaa052.

7. Whiteside, T. L. Exosomes carrying immunoinhibitory proteins and their role in cancer. Clin. Exp. Immunol. 189, 259-267 (2017).

8. Marleau, A. M., Chen, C. S., Joyce, J. A. \& Tullis, R. H. Exosome removal as a therapeutic adjuvant in cancer. J. Transl. Med. 10, 134 (2012).

9. Galieva, L. R., James, V., Mukhamedshina, Y. O. \& Rizvanov, A. A. Therapeutic Potential of Extracellular Vesicles for the Treatment of Nerve Disorders. Front. Neurosci.13, (2019).

10. Kim, H. et al. Engineered extracellular vesicles and their mimetics for clinical translation. Methods. 177, 80-94 (2020).

11. Savina, A., Furlán, M., Vidal, M. \& Colombo, M. I. Exosome Release Is Regulated by a Calciumdependent Mechanism in K562 Cells. J. Biol. Chem. 278, 20083-20090 (2003).

12. Aharon, A., Tamari, T. \& Brenner, B. Monocyte-derived microparticles and exosomes induce procoagulant and apoptotic effects on endothelial cells. Thromb. Haemost. 100, 878-885 (2008).

13. Savina, A., Furlán, M., Vidal, M. \& Colombo, M. I. Exosome Release Is Regulated by a Calciumdependent Mechanism in K562 Cells. J. Biol. Chem. 278, 20083-20090 (2003).

14. Stoeck, A. et al. A role for exosomes in the constitutive and stimulus-induced ectodomain cleavage of L1 and CD44. Biochem. J. 393, 609-618 (2006).

15. Trajkovic, K. et al. Ceramide Triggers Budding of Exosome Vesicles into Multivesicular Endosomes. Science. 319, 1244-1247 (2008).

16. Babuta, M. et al. Dysregulated Autophagy and Lysosome Function Are Linked to Exosome Production by Micro-RNA 155 in Alcoholic Liver Disease. Hepatology. 70, 2123-2141 (2019).

17. Cashikar, A. G. \& Hanson, P. I. A cell-based assay for CD63-containing extracellular vesicles.PLoS ONE14, (2019). 
18. Bilodeau, P. S., Urbanowski, J. L., Winistorfer, S. C. \& Piper, R. C. The Vps27p-Hse1p complex binds ubiquitin and mediates endosomal protein sorting. Nat. Cell Biol. 4, 534-539 (2002).

19. Wollert, T. \& Hurley, J. H. Molecular mechanism of multivesicular body biogenesis by ESCRT complexes. Nature. 464, 864-869 (2010).

20. Dores, M. R. et al. ALIX binds a YPX3L motif of the GPCR PAR1 and mediates ubiquitin-independent ESCRT-III/MVB sorting. J. Cell Biol. 197, 407-419 (2012).

21. Blanc, L. \& Vidal, M. New insights into the function of Rab GTPases in the context of exosomal secretion. Small GTPases. 9, 95-106 (2018).

22. Kalluri, R. \& LeBleu, V. S. The biology, function, and biomedical applications of exosomes. Science. 367, eaau6977 (2020).

23. Messenger, S. W., Woo, S. S., Sun, Z. \& Martin, T. F. J. A Ca2+-stimulated exosome release pathway in cancer cells is regulated by Munc13-4. J. Cell Biol. 217, 2877-2890 (2018).

24. Datta, A. et al. High-throughput screening identified selective inhibitors of exosome biogenesis and secretion: A drug repurposing strategy for advanced cancer. Sci. Rep. 8, 8161 (2018).

25. Miyanishi, M. et al. Identification of Tim4 as a phosphatidylserine receptor. Nature. 450, 435-439 (2007).

26. Skotland, T., Hessvik, N. P., Sandvig, K. \& Llorente, A. Exosomal lipid composition and the role of ether lipids and phosphoinositides in exosome biology. J. Lipid Res. 60, 9-18 (2019).

27. Nakai, W. et al. A novel affinity-based method for the isolation of highly purified extracellular vesicles. Sci. Rep. 6, 33935 (2016).

28. Porter, A. G. \& Jänicke, R. U. Emerging roles of caspase-3 in apoptosis. Cell Death Differ. 6, 99-104 (1999).

29. Li, J. et al. Identification and Characterization of 293T Cell-Derived Exosomes by Profiling the Protein, mRNA and MicroRNA Components. PLOS ONE. 11, e0163043 (2016).

30. Mizrak, A. et al. Genetically engineered microvesicles carrying suicide mRNA/protein inhibit schwannoma tumor growth. Mol. Ther. J. Am. Soc. Gene Ther. 21, 101-108 (2013).

31. Ohno, S. et al. Systemically Injected Exosomes Targeted to EGFR Deliver Antitumor MicroRNA to Breast Cancer Cells. Mol. Ther. 21, 185-191 (2013).

32. Yeo, R. W. Y. et al. Mesenchymal stem cell: An efficient mass producer of exosomes for drug delivery. Adv. Drug Deliv. Rev. 65, 336-341 (2013).

33. Sato, S. et al. EPHB2 carried on small extracellular vesicles induces tumor angiogenesis via activation of ephrin reverse signaling.JCl Insight4,.

34. Kucharzewska, P. et al. Exosomes reflect the hypoxic status of glioma cells and mediate hypoxiadependent activation of vascular cells during tumor development. Proc. Natl. Acad. Sci. 110, 73127317(2013).

35. Skog, J. et al. Glioblastoma microvesicles transport RNA and proteins that promote tumour growth and provide diagnostic biomarkers. Nat. Cell Biol. 10, 1470-1476 (2008). 
36. Good, D. J. et al. A tumor suppressor-dependent inhibitor of angiogenesis is immunologically and functionally indistinguishable from a fragment of thrombospondin. Proc. Natl. Acad. Sci. 87, 66246628(1990).

37. Lai, R. C. et al. Exosome secreted by MSC reduces myocardial ischemia/reperfusion injury. Stem Cell Res. 4, 214-222 (2010).

38. Wang, J., Bonacquisti, E. E., Brown, A. D. \& Nguyen, J. Boosting the Biogenesis and Secretion of Mesenchymal Stem Cell-Derived Exosomes.Cells9, (2020).

39. Niu, Y. et al. PTEN Activation by DNA Damage Induces Protective Autophagy in Response to Cucurbitacin B in Hepatocellular Carcinoma Cells. Oxid. Med. Cell. Longev. 2016, (2016).

40. Kang, M. H. \& Reynolds, C. P. Bcl-2 Inhibitors: Targeting Mitochondrial Apoptotic Pathways in Cancer Therapy. Clin. Cancer Res. Off. J. Am. Assoc. Cancer Res. 15, 1126-1132 (2009).

41. Rong, Y. \& Distelhorst, C. W. Bcl-2 Protein Family Members: Versatile Regulators of Calcium Signaling in Cell Survival and Apoptosis.22(2007).

42. Siddiqui, W. A., Ahad, A. \& Ahsan, H. The mystery of BCL2 family: Bcl-2 proteins and apoptosis: an update. Arch. Toxicol. 89, 289-317 (2015).

43. Tsutsui, T. et al. Glioma-derived extracellular vesicles promote tumor progression by conveying WT1. Carcinogenesis bgaa052(2020) doi:10.1093/carcin/bgaa052.

\section{Tables}

Table 1. Activity of EV regulators in U87MG cells. 


\begin{tabular}{|c|c|c|c|c|}
\hline Name & CAS No. & $\begin{array}{l}\mathrm{CD} 9^{+}-\mathrm{EV} \text { s in ELISA } \\
(\%)\end{array}$ & $\begin{array}{l}\text { CD63+-EVs in ELISA } \\
(\%)\end{array}$ & $\begin{array}{l}\text { EV particles in NTA } \\
(\%)\end{array}$ \\
\hline DMSO & $67-68-5$ & 100.0 & 100.0 & 100.0 \\
\hline AA2 & 79183-19-0 & 33.6 & 54.7 & 64.9 \\
\hline Amlodipine & $88150-42-9$ & 197.5 & 213.8 & 170.8 \\
\hline Osimertinib & $\begin{array}{l}1421373- \\
65-0\end{array}$ & 174.9 & 147.9 & 157.7 \\
\hline $\begin{array}{l}\text { Cucurbitacin } \\
\text { B }\end{array}$ & $6199-67-3$ & 325.6 & 202.7 & 144.6 \\
\hline Doramectin & $\begin{array}{l}117704-25- \\
3\end{array}$ & 251.3 & 212.9 & 168.6 \\
\hline Gossypol & $303-45-7$ & 571.9 & 295.1 & 88.4 \\
\hline HA14-1 & $65673-63-4$ & 227.6 & 154.2 & 123.4 \\
\hline Miltefosine & $58066-85-6$ & 295.5 & 134.1 & 245.2 \\
\hline Obatoclax & $\begin{array}{l}803712-67- \\
6\end{array}$ & 251.3 & 390.8 & 114.7 \\
\hline
\end{tabular}

Due to technical limitations, table 2 is only available as a download in the Supplemental Files section.

Table 3. Concentrations of AA2, cucurbitacin B, gossypol, or obatoclax in different cells. 


\begin{tabular}{|lllll|}
\hline Cells & AA2 & $\begin{array}{l}\text { Cucurbitacin B } \\
(\mu \mathrm{M})\end{array}$ & $\begin{array}{l}\text { Gossypol } \\
(\mu \mathrm{M})\end{array}$ & $\begin{array}{l}\text { Obatolax } \\
(\mu \mathrm{M})\end{array}$ \\
\hline THP1 & 10 & 0.1 & 1 & 0.1 \\
\hline SW620 & 10 & 3 & 3 & 0.03 \\
\hline U87MG & 10 & 1 & 10 & 1 \\
\hline SW480 & 10 & 0.3 & 3 & 0.1 \\
\hline HEK293T & 3 & 1 & 0.3 & 0.03 \\
\hline HCT116 & 10 & 3 & 3 & 0.03 \\
\hline Jurkat & 1 & 0.01 & 1 & 0.03 \\
\hline EL4 & 0.3 & 0.03 & 1 & 0.01 \\
\hline NIH/3T3 & 1 & 0.1 & 3 & 0.03 \\
\hline hMSC & n.t. & 1 & 10 & 1 \\
\hline K562 & n.t. & 0.03 & 0.3 & 0.1 \\
\hline
\end{tabular}

n.t.; not tested

Figures 


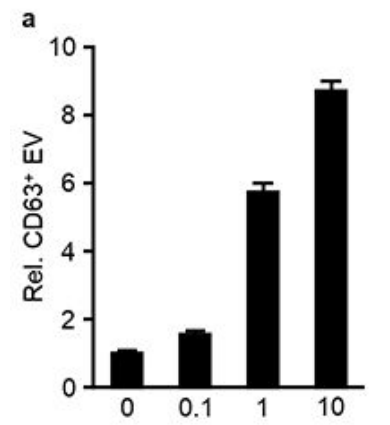

c

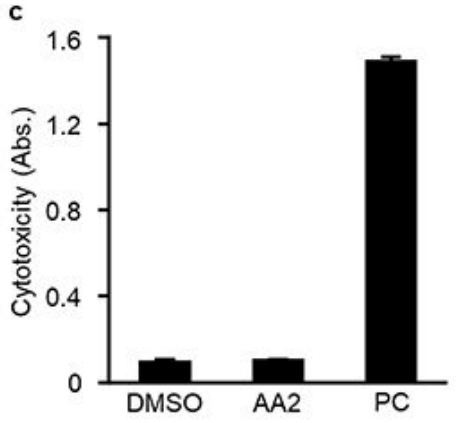

g
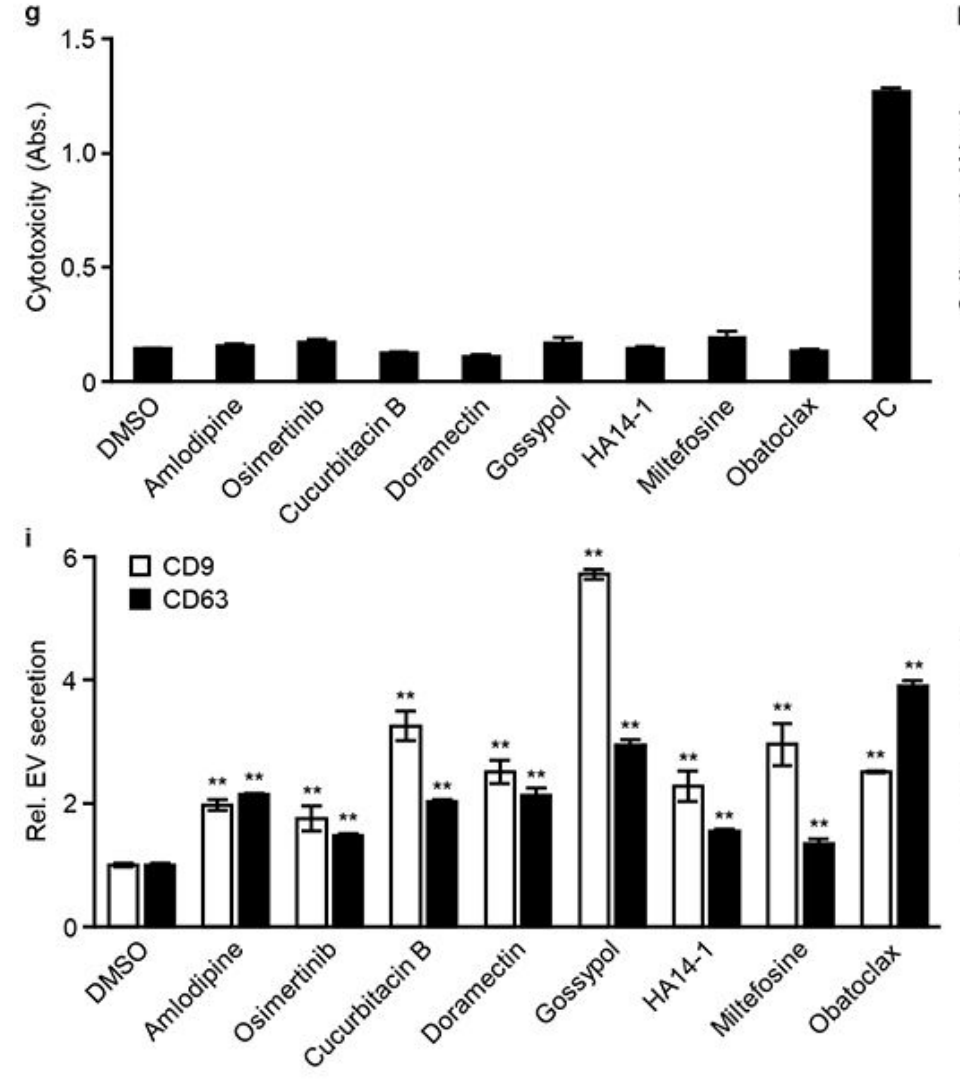

d
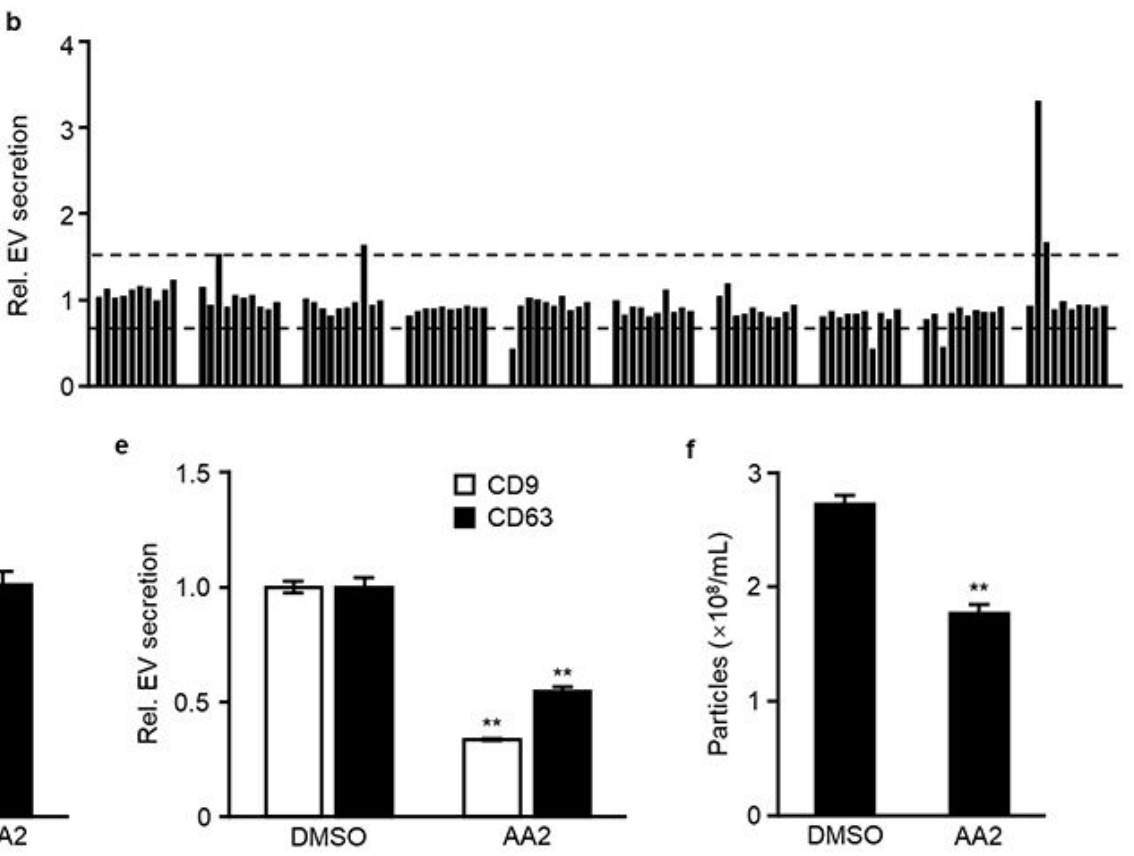

h
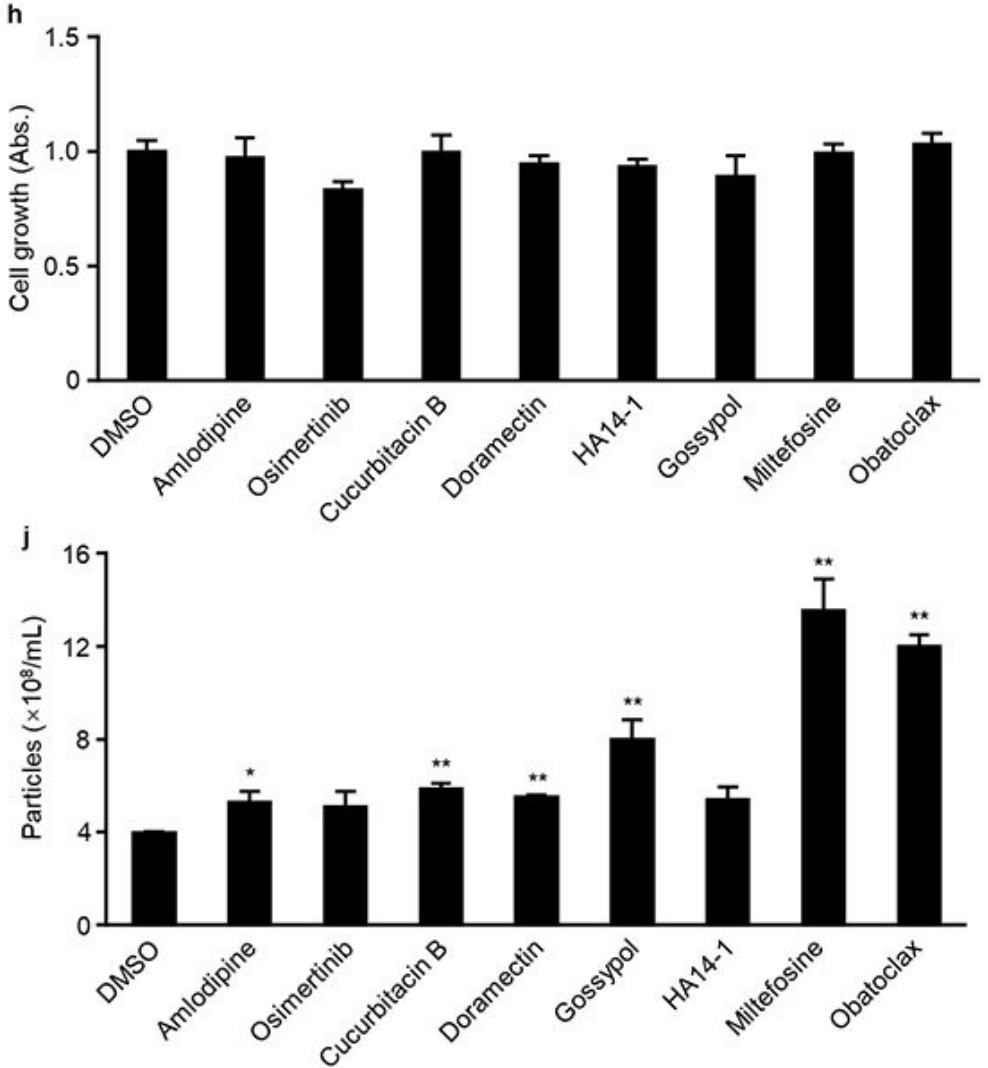

Fig. 1. Ma et. al.

\section{Figure 1}

A TIM4-affinity ELISA screen in U87MG cells identified eight activators and one inhibitor of extracellular vesicles (EVs). (a) K562 cells were stimulated for $24 \mathrm{~h}$ with 0.1 , 1, or $10 \mu \mathrm{M}$ monensin. EVs contained in the cultured supernatants were detected using TIM4-CD63 ELISA (left) and nanoparticle tracking analysis (NTA; right). (b) In the first round of screening, U87MG cells were treated with a 1,567-compound library at $0.1,1$, or $10 \mu \mathrm{M}$ for $24 \mathrm{~h}$. EVs contained in the cultured supernatants were detected using TIM4-CD63 
ELISA. A typical result is shown here. The two dashed lines represent the threshold values for activators and inhibitors, at 0.67 and 1.5 , respectively. (c-j) U87MG cells were treated with $10 \mu \mathrm{M} A A 2,7 \mu \mathrm{M}$ amlodipine, $2 \mu \mathrm{M}$ osimertinib, $1 \mu \mathrm{M}$ cucurbitacin B, $2 \mu \mathrm{M}$ doramectin, $10 \mu \mathrm{M}$ gossypol, $15 \mu \mathrm{M}$ HA14-1, 20 $\mu \mathrm{M}$ miltefosine, or $1 \mu \mathrm{M}$ obatoclax for $24 \mathrm{~h}$. Cytotoxicity and cell growth were determined using lactate dehydrogenase (LDH) $(c, g)$ and WST-8 $(d, h)$ assays. Cells treated with lysis buffer were used as a positive control (PC) in the LDH assay. Secreted EVs were detected using TIM4-CD9 or TIM4-CD63 ELISA $(e, i)$ and NTA (f, j). *; $p<0.05, * * ; p<0.01$, vs. DMSO, Student's t-test.
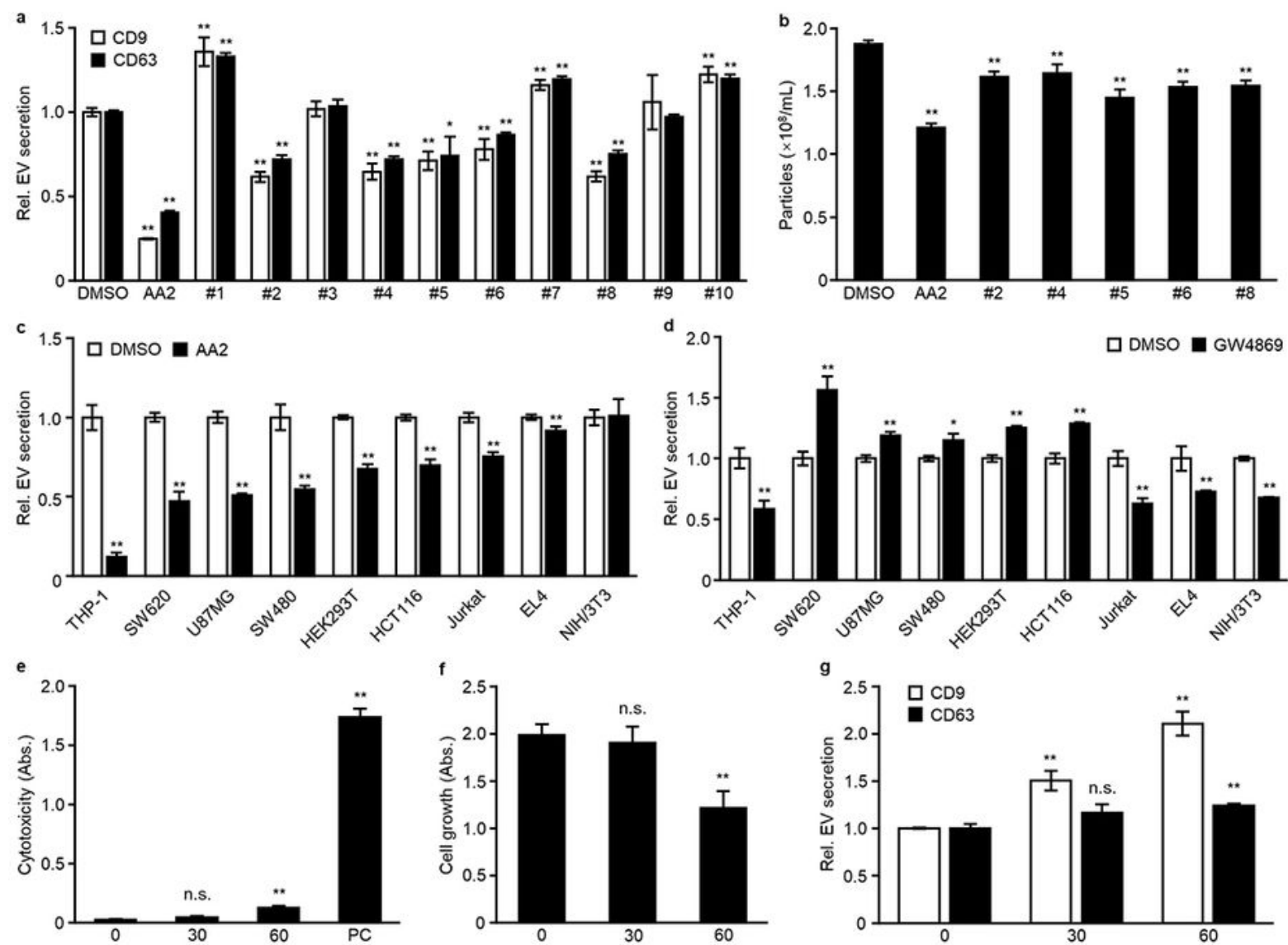

口 DMSO GW4869
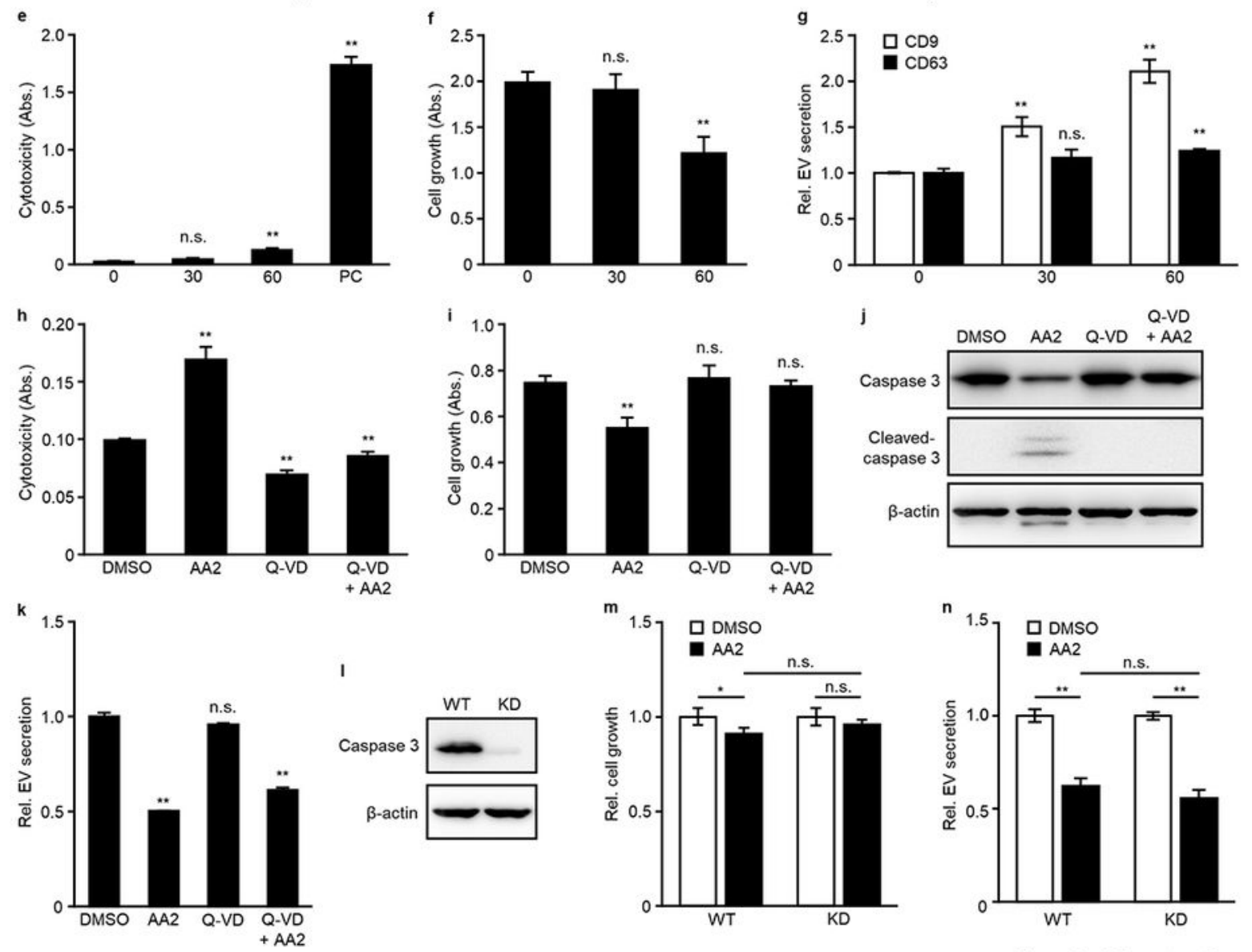

Fig. 2. Ma et. al. 
Figure 2

AA2 inhibited EV secretion independent of nSMase2 and caspase 3. (a, b) U87MG cells were treated for $24 \mathrm{~h}$ with \#1,\#7, or \#10 at $1 \mu \mathrm{M} ; \mathrm{AA2}, \# 2-\# 6, \# 8$, or \#9 at $10 \mu \mathrm{M}$, and then secreted EVs were determined using TIM4-CD9 or TIM4-CD63 ELISA (a) or NTA (b). (c, d) Cell lines were treated with AA2 as shown in Table 3 (c) or $3 \mu \mathrm{M}$ GW4869 (d) for $24 \mathrm{~h}$ and then secreted EVs were determined using TIM4 ELISA. *; $\mathrm{p}<$ 0.05 , **; $p<0.01$, vs. DMSO, Student's t-test. (e-g) U87MG cells were treated with 30 or $60 \mu \mathrm{M} M T-21$ for $24 \mathrm{~h}$. Cytotoxicity and cell growth were determined using LDH (e) and WST-8 (f) assays. Secreted EVs were determined using TIM4-CD9 or TIM4-CD63 ELISA (g). (h-k) Jurkat cells were pre-treated with 0 or 50 $\mu \mathrm{M} Q-V D$ for $3 \mathrm{~h}$, and then with 0 or $5 \mu \mathrm{M} \mathrm{AA} 2$ for $24 \mathrm{~h}$. Cytotoxicity and cell growth were determined using LDH (h) and WST-8 (i) assays. (j) The cells were lysed and immunoblotted with anti-caspase 3 , anticleaved-caspase 3 , or anti- $\beta$-actin antibody. (k) Secreted EVs were determined using TIM4-CD81 ELISA. (I) HEK293T WT or CASP3 KD cells were lysed and immunoblotted with anti-caspase 3 or anti- $\beta$-actin antibody. $(m, n)$ HEK293T WT or CASP3 KD cells were treated with $3 \mu \mathrm{M}$ AA2 for $24 \mathrm{~h}$. Cell growth was determined using a WST-8 assay $(\mathrm{m})$ and secreted EVs were determined in TIM4-CD81 ELISA (n). *; $<<$ $0.05, * * ; p<0.01$, vs. AA2, Student's t-test.

a

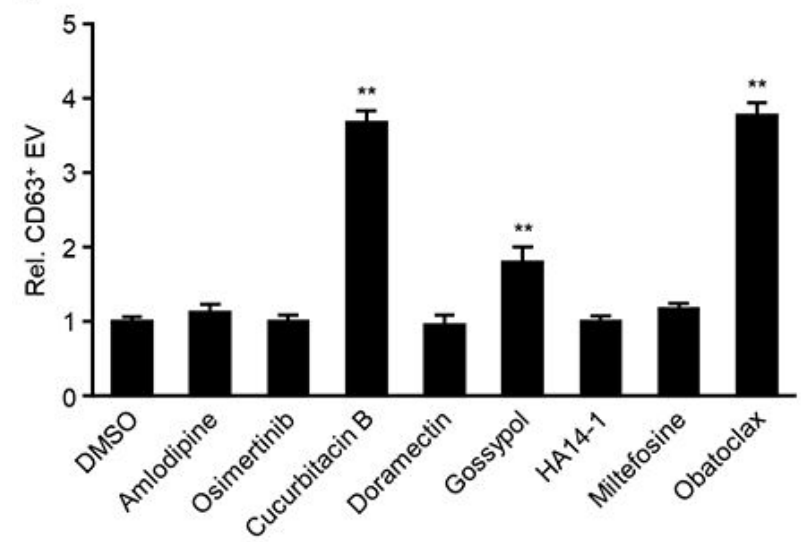

b
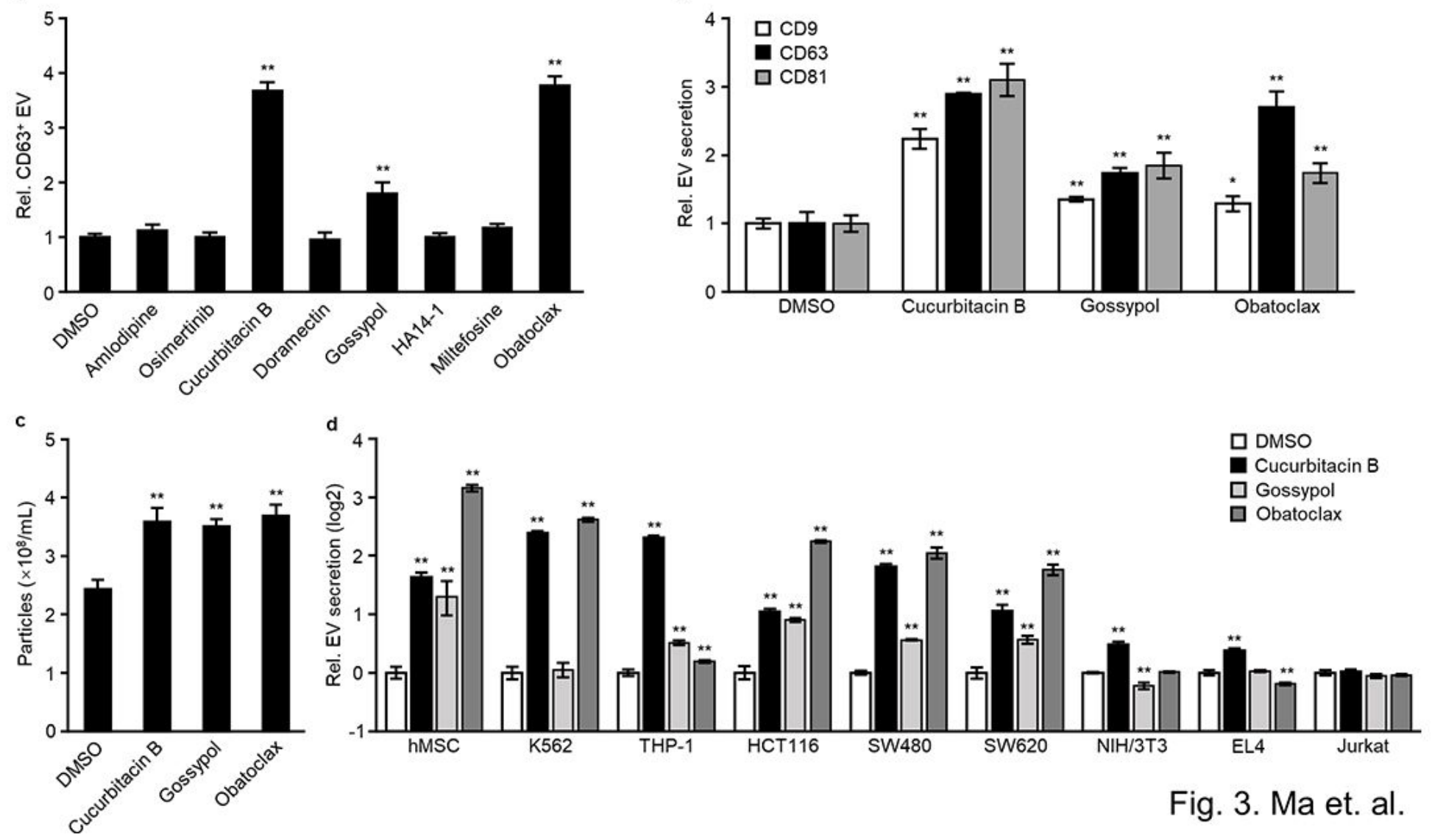

Fig. 3. Ma et. al.

Figure 3

Cucurbitacin B, gossypol, and obatoclax induced EV secretion in a variety of cells. (a-c) HEK293T cells were treated with $1 \mu \mathrm{M}$ amlodipine, $0.3 \mu \mathrm{M}$ osimertinib, $1 \mu \mathrm{M}$ cucurbitacin $\mathrm{B}, 1 \mu \mathrm{M}$ doramectin, $0.3 \mu \mathrm{M}$ gossypol, $10 \mu \mathrm{M}$ HA14-1, $10 \mu \mathrm{M}$ miltefosine, or $0.03 \mu \mathrm{M}$ obatoclax for $24 \mathrm{~h}$ and then secreted EVs were 
determined using a TIM4-CD63 ELISA (a), TIM4-CD9, TIM4-CD63, TIM4-CD81 ELISA (b) or NTA (c). (d) Cell lines were treated with cucurbitacin $B$, gossypol, or obatoclax at the concentrations shown on Table 3 for $24 \mathrm{~h}$, and then secreted EVs were determined using a TIM4 ELISA. *; $p<0.05, * * ; p<0.01$, vs. DMSO, Student's t-test.
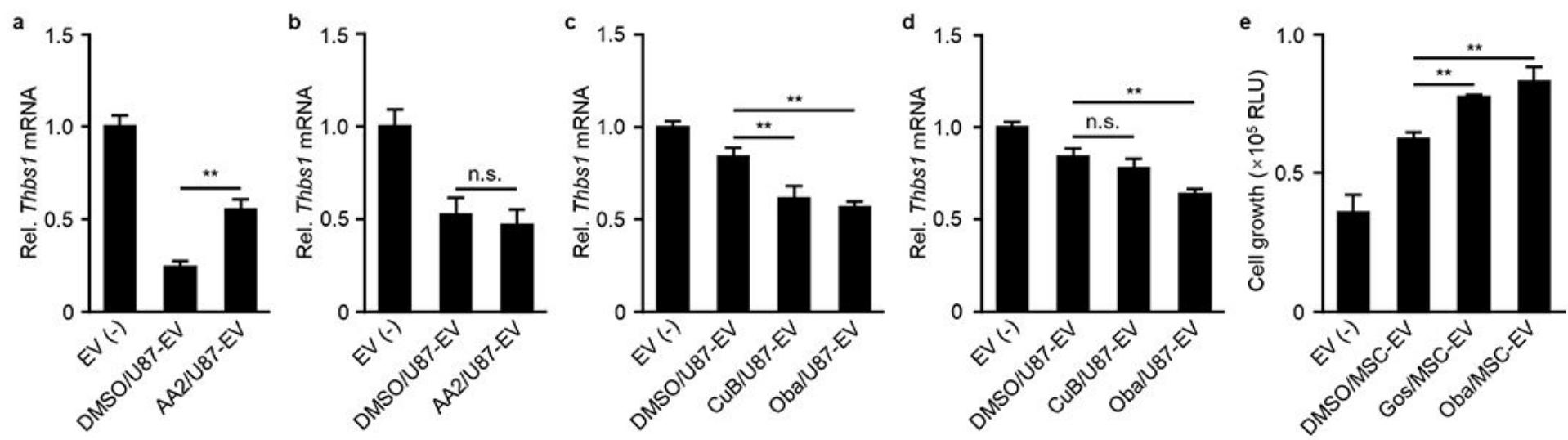

Fig. 4. Ma et. al.

\section{Figure 4}

AA2, cucurbitacin B, gossypol, and obatoclax can regulate EV bioactivity in vitro. DMSO/U87-, AA2/U87-, $\mathrm{CuB} / \mathrm{U} 87$-, or Oba/U87-EVs were isolated from the cultured supernatant of U87MG cells following treatment for 2 days with DMSO, AA2, cucurbitacin B, or obatoclax. MG6 cells were stimulated with EVs isolated from the same number of U87MG cells (a; $1 \times 107$ cells, $c ; 3 \times 106$ cells) or the same number of EV particles (b; $1 \times 1010$ particles, d; $3 \times 109$ particles) for $12 \mathrm{~h}$. Total RNA was extracted from MG6 and the expression of Thbs1 was detected via RTqPCR. *; $p<0.05$, **; $p<0.01$, n.s.; not significant, vs. DMSO/U87-EV, Student's t-test. (e) DMSO/MSC -, Gos/MSC -, or Oba/MSC-EVs were isolated from the cultured supernatant of hMSCs following treatment for 2 days with DMSO, gossypol, or obatoclax. H9C2 cells pre-treated with $1 \mathrm{mM} \mathrm{CoCl} 2$ were stimulated with the EVs isolated from $4 \times 105$ hMSCs for 4 days. Cell growth was measured via a luminescent cell viability assay. $* ; p<0.05, * * ; p<0.01$, n.s.; not significant, vs. DMSO/MSC-EV, Student's t-test.

\section{Supplementary Files}

This is a list of supplementary files associated with this preprint. Click to download.

- SupplemenntTIM4ELISAscreening210130.docx

- TIM4ELISAscreeningMa20210115Table2.docx 